\title{
HOT-ELECTRON NOISE FEATURES IN SILICON CRYSTALS OPERATING UNDER PERIODIC SIGNALS
}

\author{
D. Persano Adorno, M.A. Lodato, N. Pizzolato, and B. Spagnolo \\ Department of Physics and Chemistry, Group of Interdisciplinary Physics and CNISM, University of Palermo, \\ Viale delle Scienze - Ed. 18, 90128 Palermo, Italy \\ E-mail: dominique.persanoadorno@unipa.it
}

Received 18 November 2013; accepted 4 December 2013

\begin{abstract}
We study the intrinsic noise in n-type Si crystals operating under high-frequency periodic electric fields. To simulate the dynamics of electrons in the bulk, by taking into account the main details of band structure, scattering processes, as well as heating effects, a Monte Carlo approach is used. The noise properties are investigated by computing the velocity fluctuations correlation function, its spectral density, and the total noise power for different values of the amplitude and frequency of the driving field. We show that the noise features are significantly affected by the electric field amplitude and frequency and discuss their peculiarities in comparison with those exhibited in the static field case. We find the integrated spectral density, i. e. the total noise power, monotonically reducing its value with the increase of the field frequency, for each amplitude of the applied field. These results can be considered a first step towards a full understanding of the physical characteristics of electronic noise in Si devices, driven by periodic electric fields, relevant, for example, for harmonic generation purposes.
\end{abstract}

Keywords: electronic noise, Monte Carlo simulation, high-frequency electric fields

PACS: 05.10.Ln , 72.70.+m, 05.40.Ca, 72.20.-i

\section{Introduction}

The requirement of high integration capability of microelectronic and optoelectronic components has given rise to the development of more and more advanced fabrication techniques with increasing level of accuracy. Such a miniaturization process causes charge carriers to be driven by very intense electric field strengths, typically of the order of $\mathrm{kV} / \mathrm{cm}$ or tens of $\mathrm{kV} / \mathrm{cm}$, also at ordinary voltages. Then, electron transport takes place in a strongly nonlinear regime. For this reason, the Monte Carlo method has been largely used to investigate and characterize the transport properties of hot electrons in semiconductor structures in the presence of electric and/or magnetic fields (see e. g. [1-3]).

The presence of intrinsic noise limits the performance of electronic devices and affects the sensitivity of semiconductor-based circuits. Moreover, an intense periodic electric field can deeply modify the spectral density of electron fluctuations with respect to the case in which a static electric field is applied. For this reason, several studies have analysed the electronic noise in devices operating under static and large-signal periodic conditions [4-16. In spite of this, so far little has been done to investigate elec- tron noise properties in silicon structures operating under oscillating electric fields. Previous studies on the electron velocity fluctuations in GaAs crystals, driven by intense periodic electric fields, have shown that the total noise power depends on both the amplitude and the frequency of the excitation signal [14, 15]. Moreover, the possibility to suppress the diffusion noise in GaAs bulks, by adding a Gaussian correlated or dichotomous fluctuating contribution to the driving periodic electric field, has been recently found [17-19].

In this paper, we focus on electron transport properties in n-silicon crystals embedded in high frequency alternating electric fields. We use a Monte Carlo method to simulate the dynamics of electrons in the bulk and calculate relevant quantities, such as the electron velocity auto-correlation function, the spectral density of fluctuations, and the variance, i. e. the total noise power. The noise features of hot-carriers driven by the periodic electric field are compared with those obtained in the presence of a static field, with the aim to investigate the modifications of the electronic noise spectra induced by the frequency and the strength of the applied field.

The paper is organized as follows: in Section 2 we briefly present the statistical quantities used to 
characterize the intrinsic electronic noise and give some details on the Monte Carlo algorithm used for our simulations; in Section 3 the numerical results are presented and discussed. Final comments and conclusions are given in Section 4.

\section{Noise calculation method and Monte Carlo procedure}

The motion of electrons in semiconductor crystals, in the presence of a driving electric field, is characterized by an average velocity that depends on the amplitude and the frequency of the applied field. The fluctuations of the drift velocity with respect to the statistical average $\langle v(t)\rangle$ correspond to the intrinsic noise of the system. Therefore, the stochastic properties of electron transport can be characterized by analyzing the fielddependence of the velocity autocorrelation function and the spectral density of velocity fluctuations.

If the applied field $E_{0}$ is static, the correlation function $C_{\delta v \delta v}(\tau)$ of the velocity fluctuations can be calculated as

$$
C_{\delta v \delta v}(\tau)=\left\langle\delta \mathrm{v}\left(t^{\prime}\right) \delta v\left(t^{\prime}+\tau\right)\right\rangle,
$$

where $\tau$ is the correlation time and the brackets $\langle\ldots\rangle$ indicate ensemble average. Due to the WienerKhintchine theorem, the spectral density of the velocity fluctuations may be computed as the Fourier transform of $C_{\delta v \delta v}(\tau)$.

If the semiconductor bulk is driven by a periodic electric field with amplitude $E$ and frequency $f$,

$$
E(t)=E \cos (2 \pi f t),
$$

the velocity autocorrelation function $C_{\delta v \delta v}(t, \tau)$ can be represented as a function of two relevant times

$$
\begin{aligned}
& C_{\delta v \delta v}(t, \tau)=\langle v(t-\tau / 2) v(t+\tau / 2)\rangle- \\
& \langle v(t-\tau / 2)\rangle\langle v(t+\tau / 2)\rangle,
\end{aligned}
$$

in which $\tau$ is the correlation time and the average is done over a sequence of equivalent time instants $t=s+m T$, with $s$ belonging to the time interval $[0, T]$ ( $T$ is the field period) and $m$ is an integer [11]. This two-time symmetric correlation function eliminates any regular contribution and describes only the fluctuating part of $v(t)$. By averaging over the whole set of values of $t$ within the period $T$, the velocity autocorrelation function becomes

$$
C_{\delta v \delta v}(\tau)=\frac{1}{T} \int_{0}^{T} C_{\delta v \delta v}(t, \tau) \mathrm{d} t
$$

The transport of the electrons in the Si bulk is simulated by using a single-particle Monte Carlo algorithm, which follows the standard procedure described in Ref. [20]. The code includes the scattering by acoustic phonons, by employing a deformation potential, and the interaction with the ionized impurities, via the Brooks-Herring approximation. Moreover, the intervalley scattering is taken into account by considering six types of optical phonons, three f-type (transitions between perpendicular valleys) and three g-type (transitions between parallel valleys). The first-order time-dependent perturbation theory is used to calculate the scattering probabilities and the scattering events are considered instantaneous. We assume field-independent scattering probabilities; accordingly, the influence of the external fields is only indirect through the fieldmodified electron velocities. The model for the conduction band of silicon and the parameters of the scattering mechanisms are the same of those reported in Ref. [21, 22]. The free electron concentration is $10^{13} \mathrm{~cm}^{-3}$. We assume that all donors are ionized and that the free electron concentration is equal to the doping concentration. All the results presented in the current paper are obtained in a steady-state regime, after that a transient time of a few ps has elapsed. Nonlinear interactions of the field with the lattice and with bound carriers are neglected.

\section{Numerical results}

\subsection{Static electric field}

Figure 1 illustrates the spectral density of electron velocity fluctuations as a function of the frequency, obtained by varying the amplitude of the static electric field $E_{0}$ in the range $2-20 \mathrm{kV} / \mathrm{cm}$, at two different lattice temperatures, namely $T=77 \mathrm{~K}$ (panel a) and $T=300 \mathrm{~K}$ (panel b). When the free carriers inside the semiconductor crystal are driven by a static electric field, the shape of the spectral density of electron velocity fluctuations strongly depends on the strength of the applied field. At $T=77 \mathrm{~K}$, for field amplitudes equal to $E_{0}=2 \mathrm{kV} / \mathrm{cm}$ and $E_{0}=5 \mathrm{kV} / \mathrm{cm}$, the shape of the spectral density is characterized by significantly higher values in the low-frequency region, by a characteristic peak at an intermediate frequency of $\sim 200$ and $\sim 400 \mathrm{GHz}$, respectively, and a rapid drop for higher frequencies. For field amplitudes equal to 10,15 , and $20 \mathrm{kV} / \mathrm{cm}$, the spectra are characterized by lower values in the low-frequency region and a broad peak at a frequency of $\sim 800 \mathrm{GHz}$. The peak, which could be viewed as a "natural" transition frequency of the system 

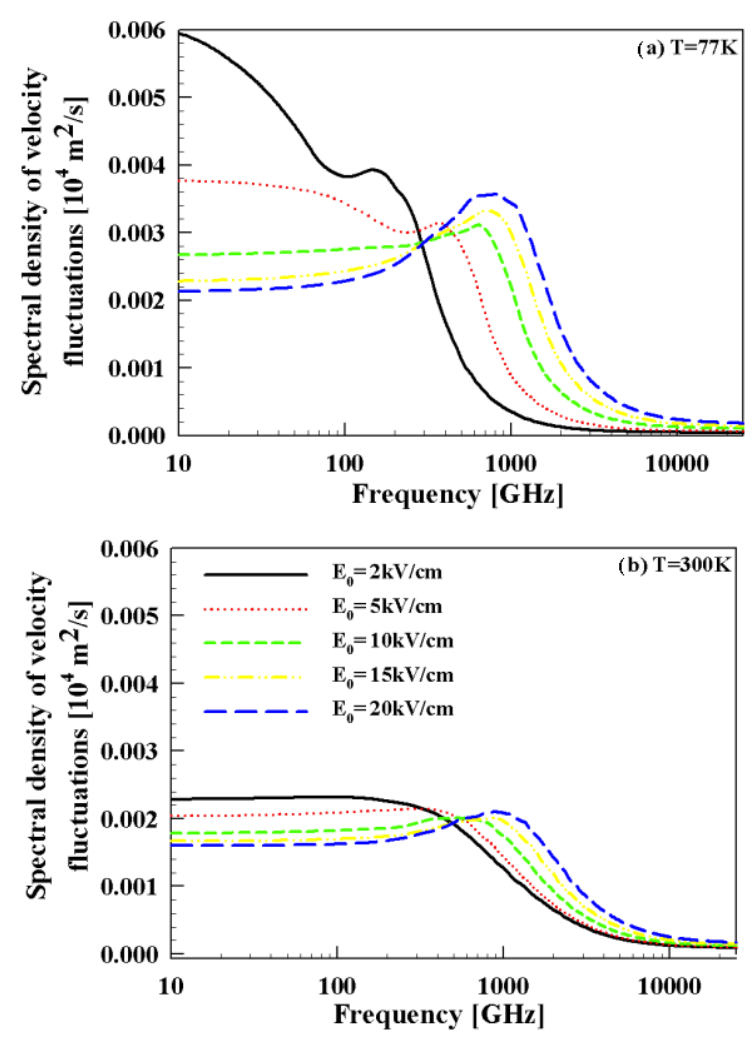

Fig. 1. Spectral density of electron velocity fluctuations obtained for different strengths of the static electric field $E_{0}$ at two different lattice temperatures: (a) $T=77 \mathrm{~K}$ and (b) $T=300 \mathrm{~K}$.

between different valleys, is found at a frequency that increases with the intensity of the applied field $E_{0}$. At $T=300 \mathrm{~K}$ (Fig. 1(b)), the spectral densities show a typical Lorentzian shape, the peaks are less pronounced, and the enhancement at low frequencies is completely absent.

The total noise power, i. e. the integrated spectral density (ISD), is shown in Fig. 2 as a function of the amplitude of the applied static field. For the two investigated lattice temperatures, the ISD shows an almost linear increasing behaviour with $E_{0}$.

It is interesting to note that, being the slope of the curve at $T=300 \mathrm{~K}$ lower than that at $T=77 \mathrm{~K}$, for field values greater than $\sim 12 \mathrm{kV} / \mathrm{cm}$, the ISD at $T=300 \mathrm{~K}$ remains lower than the values computed at $T=77 \mathrm{~K}$.

\subsection{Oscillating electric field}

In the panels of Fig. 3 we show the spectral density of electron velocity fluctuations obtained at $T=77 \mathrm{~K}$, field amplitude $E$ in the range of $2-20 \mathrm{kV} / \mathrm{cm}$ at different frequencies, namely: (a) $100 \mathrm{GHz}$, (b) $200 \mathrm{GHz}$, (c) $500 \mathrm{GHz}$, and (d) $1 \mathrm{THz}$.
For $f=100 \mathrm{GHz}$, the spectra show the characteristic shape enriched by the presence of peaks at the frequency $f$ and at its odd harmonics (up to the 11th for $E=20 \mathrm{kV} / \mathrm{cm}$ ). In the low frequency range, the spectral density monotonically decreases at the increasing of the electric field amplitude. At $f=200 \mathrm{GHz}$, the spectra obtained at $E=2 \mathrm{kV} / \mathrm{cm}$ and $E=5 \mathrm{kV} / \mathrm{cm}$ show a significant enhancement in the low-frequency region and a more pronounced peak at frequency $v=200 \mathrm{GHz}$. For stronger field amplitudes, the spectral density assumes again the diffusion shape. For $f=500 \mathrm{GHz}$ and $f=1 \mathrm{THz}$, the peaks at the harmonic frequencies are not observed. Furthermore, an enhancement in the lowfrequency region is found for $E=2 \mathrm{kV} / \mathrm{cm}, E=5 \mathrm{kV} /$ $\mathrm{cm}$ at $f=500 \mathrm{GHz}$ and for $E=10 \mathrm{kV} / \mathrm{cm}$ at $f=1 \mathrm{THz}$.

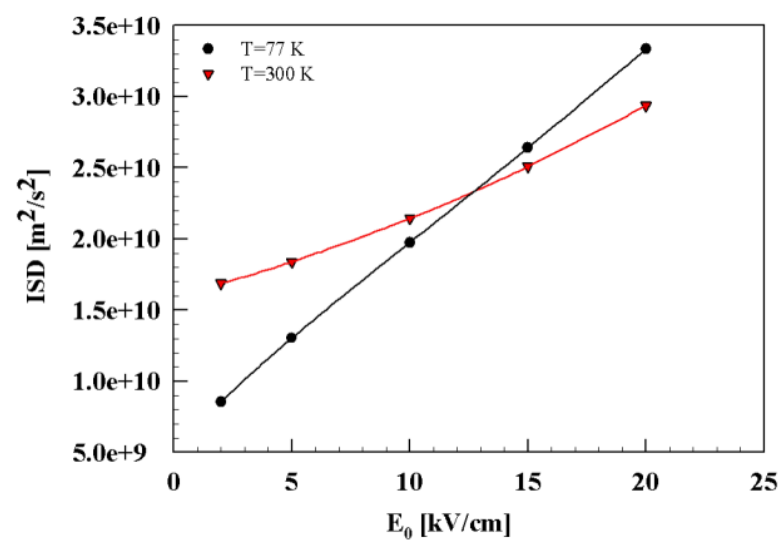

Fig. 2. ISD of electron velocity fluctuations as a function of the amplitude of the applied static field $E_{0}$ at $T=77 \mathrm{~K}$ (black line) and $T=300 \mathrm{~K}$ (red line).

In Figs. 4 and 5 we show the ISD of the electron velocity fluctuations as a function of the frequency $f$ of the driving electric field and as a function of the field amplitude $E$, respectively. The ISD obtained in the presence of an oscillating electric field is always lower than that computed in the static case for each amplitude of the applied field. It also monotonically decreases with the increasing of the frequency of the driving field. For $E=2 \mathrm{kV} / \mathrm{cm}$, it remains almost constant for frequencies of the periodic field in the range of $400<f<1000 \mathrm{GHz}$ (see Fig. (4).

Moreover, with the increasing of the amplitude of the driving field, the ISDs up to $f=200 \mathrm{GHz}$ show a linear increasing trend that remains close to the static one. Different increasing trends have been found in the case of $f=500 \mathrm{GHz}$ and $f=1 \mathrm{THz}$. The ISD obtained at $f=1 \mathrm{THz}$, for $E=20 \mathrm{kV} / \mathrm{cm}$, is reduced to about half of the value obtained in the static field case. 


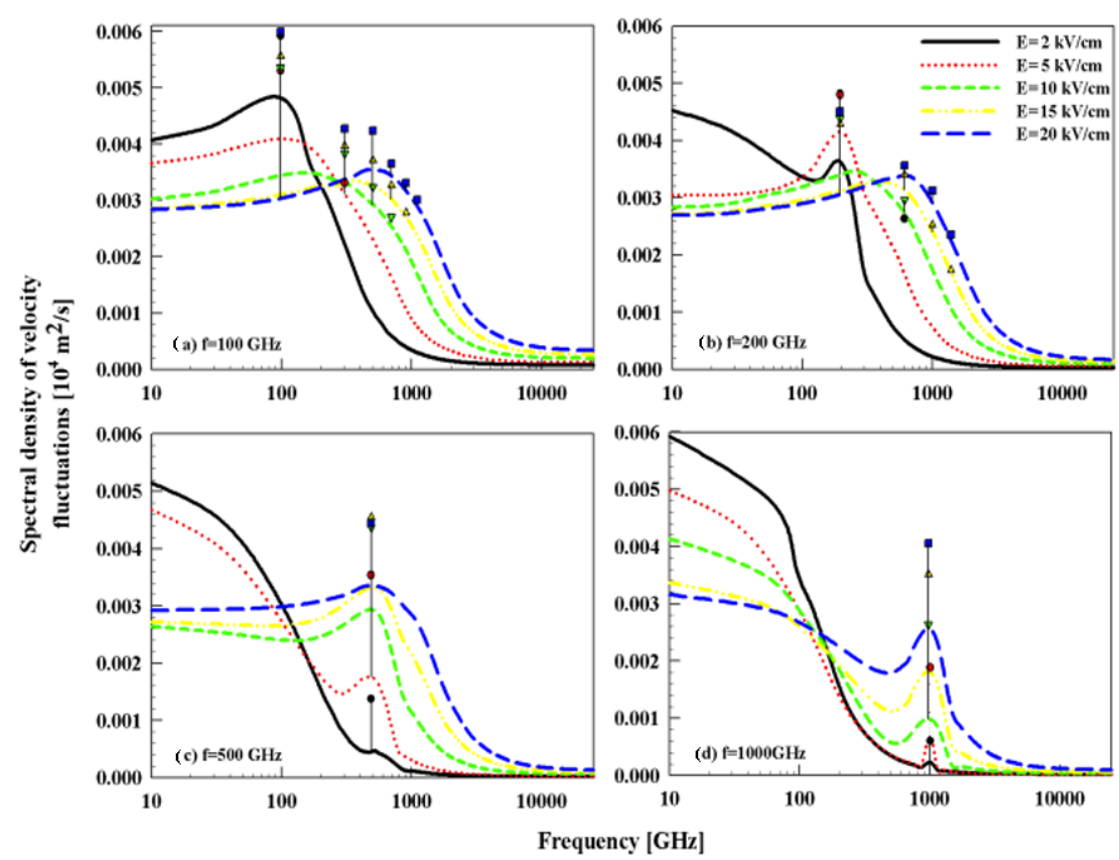

Fig. 3. Spectral density of velocity fluctuations obtained in the presence of an external periodic electric field; $T=77 \mathrm{~K}$ and (a) $f=100 \mathrm{GHz}$, (b) $f=200 \mathrm{GHz}$, (c) $f=500 \mathrm{GHz}$, and (d) $f=1 \mathrm{THz}$.

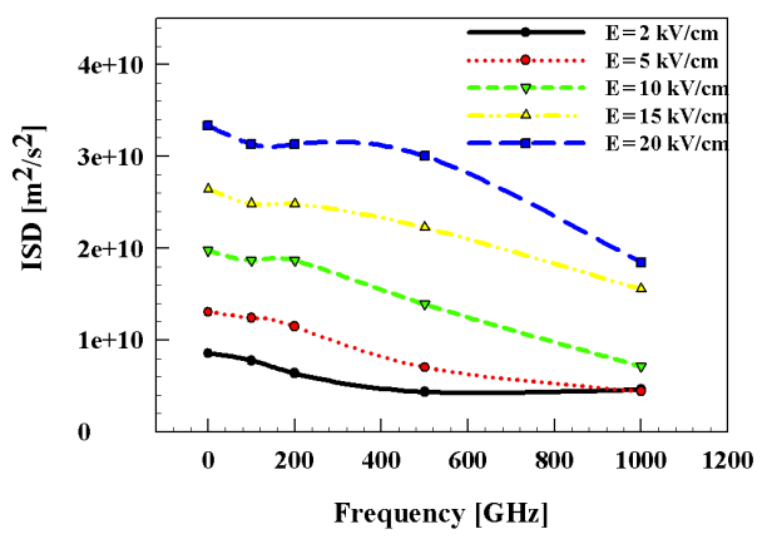

Fig. 4. ISD of electron velocity fluctuations as a function of the frequency of the periodic field at different field amplitudes $E$.

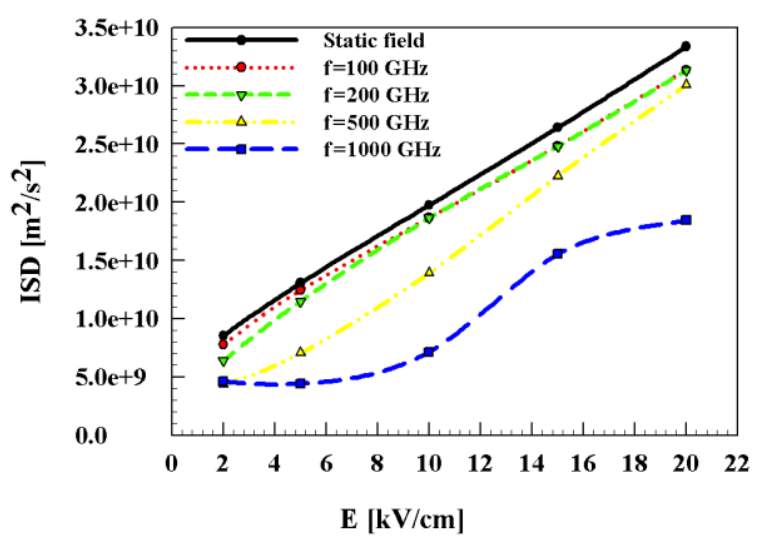

Fig. 5. ISD of the electron velocity fluctuations as a function of the amplitude $E$ of the periodic field at different values of frequency.

\section{Conclusion}

In this paper, we report the results of a Monte Carlo study on the intrinsic noise in n-type low-doped Si crystals operating under high-frequency periodic electric fields. The noise properties are investigated by computing the spectral density of the electron velocity fluctuations and the total noise power, for different values of the intensity and frequency of the driving field. The noise spectra exhibit some peculiarities, which are severely different from those obtained in the case of the static field. We have found a strong reduction in the noise level for increasing frequencies of the driving field. This effect could be a consequence of the cooling of the distribution function, but further investigations are needed to better clarify this result. Future work will be oriented towards the full comprehension and physical interpretation of the electronic noise features observed in Si devices driven by periodic electric fields, with the aim of obtaining relevant information for harmonic extraction purposes.

\section{Acknowledgements}

This work was partially supported by CNISM and by MIUR through Grant No. PON02 00355 3391233, ENERGETIC.

\section{References}

[1] W. Fawcett, A.D. Boardman, and S. Swain, J. Phys. Chem. Solids 31, 1963-1990 (1970). 
[2] A.D. Boardman, W. Fawcett, and J.G. Ruch, Phys. Stat. Sol. A 4, 133-141 (1971).

[3] C. Jacoboni and L. Reggiani, Rev. Mod. Phys. 55, 645-705 (1983).

[4] R. Fauquembergue, J. Zimmermann, A. Kaszynski, E. Constant, and G. Microondes, J. Appl. Phys. 51, 1065-1071 (1980).

[5] R.O. Grondin, P.A. Blackey, J.R. East, and E.D. Rothman, IEEE Trans. Electron Devices 28, 914-923 (1981).

[6] R. Brunetti and C. Jacoboni, Phys. Rev. B 29, 5739-5748 (1984).

[7] J.P. Nougier, IEEE Trans. Electron Devices 41, 2034-2048 (1994).

[8] L. Reggiani, P. Golinelli, L. Varani, T. González, D. Pardo, E. Starikov, P. Shiktorov, and V. Gružinskis, Microelectronics J. 28, 183-198 (1997).

[9] P. Shiktorov, E. Starikov, V. Gružinskis, L. Reggiani, L. Varani, and J.C. Vaissière, Appl. Phys. Lett. 80, 4579-4761 (2002).

[10] P. Shiktorov, E. Starikov, V. Gružinskis, M. Zarcone, D. Persano Adorno, G. Ferrante, L. Reggiani, L. Varani, J.C. Vaissière, Phys. Status Solidi A 190, 271-279 (2002).

[11]T. González, S. Pérez, E. Starikov, P. Shiktorov, V. Gružinskis, L. Reggiani, L. Varani, and J.C. Vaissière, Proc. SPIE 5113, 252-266 (2003).
[12] P. Shiktorov, E. Starikov, V. Gružinskis, S. Pérez, T. González, L. Reggiani, L. Varani, and J.C. Vaissière, Phys. Rev. B 67, 165201-165211 (2003).

[13]E.L. Pankratov and B. Spagnolo, Eur. Phys. J. B 46, 15-19 (2005).

[14] D. Persano Adorno, M.C. Capizzo, and M. Zarcone, Fluct. Noise Lett. 8, L11-L22 (2008).

[15]D. Persano Adorno, M.C. Capizzo, and N. Pizzolato, Acta Phys. Pol. A 113, 979-983 (2008).

[16] J.-F. Millithaler, L. Reggiani, J. Pousset, L. Varani, C. Palermo, J. Mateos, T. González, S. Pérez, and D. Pardo, J. Stat. Mech. Theor. Exp. 02, P02030-12 (2009).

[17] D. Persano Adorno, N. Pizzolato, and B. Spagnolo, J. Stat. Mech. Theor. Exp. 01, P01039 (2009).

[18] D. Persano Adorno, N. Pizzolato, and B. Spagnolo, Acta Phys. Pol. A 113, 985-988 (2008).

[19]D. Persano Adorno, N. Pizzolato, D. Valenti, and B. Spagnolo, Rep. Math. Phys. 70, 171-179 (2012).

[20] D. Persano Adorno, M. Zarcone, and G. Ferrante, Laser Phys. 10, 310-315 (2000).

[21] D. Persano Adorno, M. Zarcone, and G. Ferrante, Laser Phys. 11, 291-295 (2001).

[22] D. Persano Adorno, M. Zarcone, and G. Ferrante, Laser Phys. 13, 270-274(2003).

\section{KARŠTŲJŲ ELEKTRONỤ TRIUKŠMỤ SAVYBĖS SILICIO KRISTALUOSE VEIKIANT PERIODINIAMS SIGNALAMS}

D. Persano Adorno, M.A. Lodato, N. Pizzolato, B. Spagnolo

Palermo universitetas, Palermas, Italija 